\title{
Post-Malaria Neurological Syndrome - A Case of Bell's Palsy After Plasmodium Vivax Malaria
}

\section{Singh AK ${ }^{1}$, Chakraborti $S^{2}$, Subhranag $\mathbf{S}^{3}$}

${ }^{1}$ Dr. Arvind Kumar Singh, MBBS, MD, Senior Resident, Kalawati Saran Children's Hospital, Lady Hardinge Medical College, New Delhi, India, ${ }^{2}$ Dr. Snehansu Chakraborti, MBBS, MD, Professor, Department of Paediatrics, BSMC, Bankura, West Bengal, India, ${ }^{3}$ Dr. Shankha Subhra Nag, MBBS, MD, Senior Resident. Dept of Paediatrics, BC Roy Post Graduate Institute of Paediatric Sciences, Kolkata, India.

\section{Introduction}

$\mathrm{P}$ MNS is usually a short-lived, self-limiting condition with no long-term neurological sequelae. The time from eradication of systemic parasitaemia to the development of this syndrome can be up to two months ${ }^{1}$. Clinical spectrum include generalised convulsions, acute confusional state, psychosis, tremors, cerebellar ataxia, motor aphasia, generalised myoclonus, bilateral facial nerve palsy ${ }^{2,3,4}$. It generally follows after recovery from Plasmodium falciparum (P.falciparum) malaria.

\section{The Case}

A six year old male was admitted in Paediatrics department with complaints of fever for 6 days, with headache. During physical examination, he was conscious, oriented and there was mild pallor but no icterus, lymphadenopathy or cyanosis. Mild splenomegaly was present. Other systems including respiratory, cardiovascular and central nervous system were normal. His blood examination revealed: $\mathrm{Hb}: 9.2$ gm\%, total leukocyte count: 7,800/cumm, neutrophile: 56\%, lymphocyte: $40 \%$, eosinophils: $03 \%$, monocyte: $01 \%$. Peripheral smear showed presence of ring form of $P$. vivax. Rapid diagnostic test kit was used to confirm the diagnosis of $P$. vivax malaria and rule out $P$. falciparum. Patient was put on chloroquine for three days. Fever subsided after three days and patient was discharged with primaquine on $5^{\text {th }}$ day of admission. After 10 days of discharge patient presented with complaints of sudden onset of weakness in right side of face, difficulty in closing his right eyelid, deviation

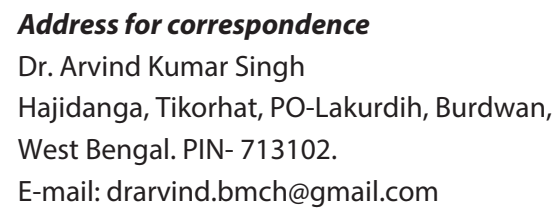

\begin{abstract}
Post-malaria neurological syndrome (PMNS) is defined as the acute onset of neurological or neuropsychiatric syndrome in a patient who had recently recovered from malaria and have negative blood film at the time of onset of neurological symptoms. It is relatively rare, with various clinical symptoms. We report first case of Bell's palsy developing on 10th day of afebrile period after successful treatment of Plasmodium vivax (P.vivax) malaria and which completely recovered in next two weeks.
\end{abstract}

of angle of mouth towards left, and drooling of saliva from right side of mouth since morning (Fig. 1). He was fully conscious and well-oriented. Examination of facial nerve revealed it to be of right sided lower motor neuron type palsy. Other cranial nerves were within normal limit. There were no cutaneous lesions of herpes zoster in the external ear canal. There was no history of fever after discharge from hospital or any known preexisting disease like diabetes mellitus. Investigation like complete blood count with peripheral blood smear, blood sugar, chest X- ray and MRI brain were normal. Patient was readmitted for observation and discharged after two days as symptoms were not progressing. No treatment was given and patient fully recovered in two weeks.

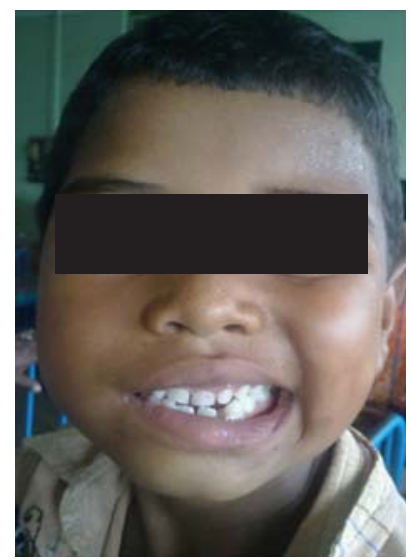

Fig 1: The patient showing features of right sided Bell's palsy

\section{How to cite this article?}

Singh AK, Chakraborti S, Subhranag S. Post-Malaria Neurological Syndrome - A Case of Bell's Palsy After Plasmodium Vivax Malaria. J Nepal Paediatr Soc 2013;33(1):66-67. 


\section{Discussion}

PMNS is defined as the acute onset of confusion, epileptic seizures, or any other neurological or psychiatric sign occurring with a latency of several days to weeks (generally within 2 months) after an episode of successfully treated $P$. falciparum malaria ${ }^{1}$. Although it generally follows after infection with $P$. falciparum but few case reports of PMNS after P.vivax malaria are available in literature, one case presented with acute disseminated encephalomyelitis like picture, second one with acute inflammatory demyelinating polyneuropathy and third with bilateral facial nerve palsy ${ }^{4}$. The prevalence of PMNS is 300 times more common in patients with severe malaria in comparison to uncomplicated malaria.

The first description of a post-infectious neurological complication of malaria was delayed cerebellar ataxia (DCA), identified in Sri Lanka in 19845,6,7. The prospective study, conducted in Vietnam and Thailand, first defining PMNS, described 22 patients, 19 adults, and 3 children, having neurological or psychiatric symptoms occurring within 2 months after an acute and cured malaria, among 18,124 patients with a treated $P$. falciparum malaria: overall incidence was 0.7 to 1.8 per 1000 in four regions were study was conducted. The syndrome was self-limiting, median duration 60 hours (range 24-240). A correlation between mefloquine therapy and PMNS was also noted ${ }^{1}$.

Schnorf and others in 1998 proposed a classification of PMNS into 3 subtypes: a mild or localized form, characterized by isolated cerebellar ataxia or postural tremor; a diffuse, but relatively mild encephalopathic form, characterized by acute confusion or epileptic seizures; and a severe, corticosteroid-responsive encephalopathy that is characterized by motor aphasia, generalized myoclonus, postural tremor, and cerebellar ataxia ${ }^{3}$.

The treatment of PMNS is mainly supportive. As reported previously, corticosteroids may be useful for patients with corticosteroids responsive encephalopathy ${ }^{3}$. However no randomized trials of corticosteroid treatment have been conducted. Response to corticosteroids provides evidence that PMNS is probably an immunologically mediated phenomenon ${ }^{8}$.

In our case, we found no evidence of cerebral Malaria. The patient developed Bell's palsy after 10 days of full recovery and repeat blood test and other relevant investigations ruled out malaria parasite in blood or any other disease process. It fully recovered after 2 weeks without any medication. These findings are consistent with PMNS. It is unlikely that the patient's symptoms were due to the toxic effect of antimalarial treatment. As the patient was treated with chloroquine and primaquine and Bell's palsy is not described as side effects of either of drug.

\section{Conclusion}

As the PMNS symptoms ranges from minor symptoms like bilateral facial nerve palsy and Bell's palsy as in this case to severe generalized encephalopathy resembling an acute disseminated encephalomyelitis (ADEM), it is important to follow up the cases and distinguish PMNS from relapse of malaria and its complications. Clinicians should be aware of PMNS so that further studies could be done to explore its pathophysiology, range of clinical spectrum, and its relationship other post-infectious neurological syndromes.

\section{References}

1. Nguyen TH, Day NP, Ly VC, Waller D, Nguyen $\mathrm{HP}$, Bethell DB, et al. Post-malaria neurological syndrome. Lancet 1996;348(9032): 917-21.

2. de Silva HJ, Gamage R, Herath HK, Abeysekera DT, Peiris JB. A delayed onset cerebellar syndrome complicating falciparum malaria. Ceylon Med $\mathrm{J}$ 1986;31(3):147-50.

3. Schnorf H, Diserens K, Schnyder H, Chofflon M, Loutan L, Chaves V, Landis T. Corticosteroidresponsive postmalaria encephalopathy characterized by motor aphasia, myoclonus, and postural tremor. Arch Neurol 1998;55(3):417-20.

4. Kochar DK, Sirohi P, Kochar SK, Bindal D, Kochar A, Jhajharia A, et al. Post-malaria neurological syndrome - a case of bilateral facial palsy after Plasmodium vivax malaria. J Vect Borne Dis 2007;44:227-29.

5. Senanayake N, de Silva HJ. Delayed cerebellar ataxia complicating falciparum malaria: a clinical study of 74 patients. J Neurol 1994;241:456-59.

6. De Silva HJ. Delayed Cerebellar ataxia: a new complication of falciparum malaria? BMJ 1987;294:1612.

7. Senanayake N. Delayed cerebellar ataxia: a new complication of falciparum malaria? BMJ 1987;294:1253-254.

8. de Silva $\mathrm{HJ}$, Hoang P, Dalton $\mathrm{H}$, de Silva NR, Jewell DP, Peiris JB. Immune activation during cerebellar dysfunction following Plasmodium falciparum malaria. Trans R Soc Trop Med Hyg 1992;86:12931. 